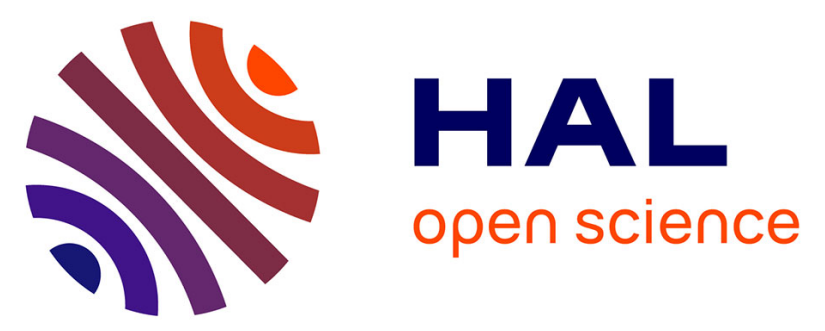

\title{
Translation of Simultaneous Vessel Wall Motion and Vectorial Blood Flow Imaging in Healthy and Diseased Carotids to the Clinic: a Pilot Study
}

Vincent Perrot, Ingvild Kinn Ekroll, Jørgen Avdal, Lars Mølgaard Saxhaug, Håvard Dalen, Didier Vray, Lasse Løvstakken, Herve Liebgott

\section{To cite this version:}

Vincent Perrot, Ingvild Kinn Ekroll, Jørgen Avdal, Lars Mølgaard Saxhaug, Håvard Dalen, et al.. Translation of Simultaneous Vessel Wall Motion and Vectorial Blood Flow Imaging in Healthy and Diseased Carotids to the Clinic: a Pilot Study. IEEE Transactions on Ultrasonics, Ferroelectrics and Frequency Control, 2020. hal-03024611

\section{HAL Id: hal-03024611 \\ https://hal.science/hal-03024611}

Submitted on 25 Nov 2020

HAL is a multi-disciplinary open access archive for the deposit and dissemination of scientific research documents, whether they are published or not. The documents may come from teaching and research institutions in France or abroad, or from public or private research centers.
L'archive ouverte pluridisciplinaire $\mathbf{H A L}$, est destinée au dépôt et à la diffusion de documents scientifiques de niveau recherche, publiés ou non, émanant des établissements d'enseignement et de recherche français ou étrangers, des laboratoires publics ou privés. 


\title{
Translation of Simultaneous Vessel Wall Motion and Vectorial Blood Flow Imaging in Healthy and Diseased Carotids to the Clinic: a Pilot Study
}

\author{
Vincent Perrot*, Ingvild Kinn Ekroll ${ }^{\dagger \dagger}$, Jørgen Avdal ${ }^{\dagger}$, Lars Mølgaard Saxhaug ${ }^{\dagger}$, Håvard Dalen ${ }^{\dagger} \S$, Didier Vray*, \\ Lasse Løvstakken ${ }^{\dagger}$, Hervé Liebgott* \\ ${ }^{*}$ CREATIS, Univ Lyon, INSA Lyon, UCBL, UJM Saint-Étienne, CNRS UMR 5220, Inserm U1206, Lyon, France \\ ${ }^{\dagger}$ CIUS and Department of Circulation and Medical Imaging, NTNU, Trondheim, Norway \\ ${ }_{\ddagger}^{\ddagger}$ Kirurgisk klinikk, St. Olavs Hospital HF, Trondheim, Norway \\ $\S$ Clinic of Cardiology, St. Olav's University Hospital, Trondheim, Norway \\ IDepartment of Medicine, Levanger Hospital, Nord-Trøndelag Hospital Trust, Levanger, Norway
}

\begin{abstract}
This study aims to investigate the clinical feasibility of simultaneous extraction of vessel wall motion and vectorial blood flow at high frame rates for extraction of clinical markers. If available in the clinic, such a technique would allow better estimation of plaque vulnerability and evaluation of overall arterial health of patients. In this study, both healthy and patient volunteers were recruited and scanned using a plane-wave acquisition scheme that provided a dataset of 43 carotid recordings in total. The vessel wall motion is extracted based on the complex autocorrelation of the signals received, while the vector flow is extracted using the transverse oscillation technique. Wall motion and vector flow are extracted at high frame rates, which allows visual appreciation of tissue movement and blood flow simultaneously and with fine temporal resolution. Several clinical markers are extracted, along with p-values. From all of the potential markers, young healthy volunteers have smaller artery diameter $(7.32 \mathrm{~mm})$ compared to diseased patients $(9.56 \mathbf{~ m m}), \mathbf{6 6} \%$ of diseased patients have backflow, a carotid with a pulse wave velocity extracted from the wall velocity that is greater than $7 \mathrm{~m} / \mathbf{s}$ is always a diseased vessel, and the peak wall shear rate decreases as the risk increases. Based on both the pathological markers and the visual inspection of tissue motion and vector flow, we conclude that the clinical feasibility of this approach is demonstrated. Larger and more disease-specific studies using such an approach will lead to better understanding and evaluation of vessels, which can translate to future use in the clinic.
\end{abstract}

Index Terms-vessel wall motion, vectorial flow, arterial properties, high frame rate, clinical study, carotid

\section{INTRODUCTION}

$\mathbf{U}$ LTRASOUND has been used with Doppler to assess cardiovascular properties since the 1950s [1], and more broadly through the 1970s [2]. Nowadays, ultrasound imaging is widely used with Doppler for routine check-ups and for evaluation of vessel disease, such as atherosclerosis

Correspondence e-mail: vincent.perrot@polymtl.ca. Vincent Perrot is now with the Engineering Physics Department, Polytechnique Montréal, Canada. This work has been performed within the framework of the LABEX CELYA (ANR-10-LABX-0060) and LABEX PRIMES (ANR-10-LABX-0063) of Université de Lyon, within the program "Investissements d'Avenir" (ANR-16-IDEX-0005) operated by the French National Research Agency (ANR). and aneurysms [3]. Vessel diseases alter the geometry of the arterial system, which impacts on the flow circulation and can create turbulent and complex flow patterns. These characteristics can be assessed through ultrasound imaging with Doppler techniques. These diseases, as well as aging and the overall health of a patient, also influence the vessel wall motion and the stiffness of the vessels [4].

Tissue motion has been studied and shown to be related to the biomechanical and geometric properties of the arterial system [4]. In clinics, pulse wave velocity (PWV) is used as a clinical marker [5] as it is related to Young's modulus through the Moens-Korteweg equation [6], [7]. PWV is used as a gold standard measurement, according to the foot-to-foot technique, which consists of detection of the time shift of the pressure waves between two distinct vessels [8], [9]. Unfortunately, this approach can only provide an overall estimate with imprecise distance measurements, and it does not reflect local vessel stiffness. In the research setting at high frame rates, PWV can be extracted from the wall velocity and it represents a robust index [10] that is directly linked to arterial stiffness [4]. Recently, PWV has been used as a local marker of pathology and it has been shown to be feasible and of particular interest [11], [12]. For flow markers, it has been shown that a low wall shear rate (WSR) can facilitate the development of plaques, whereas high WSR after plaque development can increase the risk of inflammation and rupture [13]. As the wall and flow patterns are both related to the characteristics of vessels, an imaging technique that can provide information from both of these media simultaneously should be relevant for characterization of vessel diseases. Such a technique should provide clinical markers from the vessel wall and blood flow independently, and wall/flow markers will also reflect the inherent connection between these two media. As wall motion and blood flow are inherently linked, having both measurements simultaneously from a large region of interest should be relevant for clinicians, such as to quantify the vulnerability of plaques. A rigid plaque coupled with turbulent flow represents high 
risk of disruption [14]. By obtaining more information from simultaneous measurements, we aim for better detection, more precise diagnosis, and improved monitoring of vessel diseases.

However, in clinical practice, only 1D Doppler spectrum and color Doppler flow estimates at relatively low frame rates are used, as recommended in the current guidelines [15], [16]. Consequently, most clinical studies are based on 1D flow imaging only, and ultrasound acquisitions are performed using a relatively low frame rate (below 100 images per second). Color Doppler is used for qualitative detection of abnormal flow, while the Doppler spectrum is used for quantification and requires an angle correction to provide a correct Doppler velocity [17]. Consequently, an accurate estimate needs a beam-to-flow angle below $60^{\circ}$ [17], which can be difficult in clinical cases. To overcome this angle correction, a cross-beam Doppler approach can be used [18], although this can result in considerable errors in estimates [19]. Also, geometric spectral broadening strongly affects estimates, with typical errors greater than 20\% [20]. Meanwhile, 2D vector flow, which can provide all of the velocity components in the acquisition plane at each measurement point and at high frame rates (above 1000 images per second), has been under development for years in a research setting [21], [22], and has been shown to describe complex flow patterns that occur in the carotid bulb [23].

To obtain vector velocity fields, a technique that can yield $2 \mathrm{D}$ vector flow, known as transverse oscillation (TO), was developed [24], which can be used in combination with high frame rate ultrasound sequences. This approach is based on the creation of a lateral oscillation in ultrasound images by modifying the lateral point spread function of the system. This method can be used for transmission, receiving, or post-processing by adaptive filtering. More recently, TO has been extended to tissue motion estimation [25], and validated for simultaneous extraction of wall and flow motion using both phantoms [26] and the common carotid artery of healthy volunteers [27]. Moreover, TO for flow characterization has already been used and validated in pre-clinical and small clinical studies. Notable examples here are: volume flow estimation in the carotid [28] and the arteriovenous fistula [29], velocity and volume flow estimation in the ascending aorta [30], and flow complexity assessment in the carotid [23]. Recently, TO has been compared to spectral Doppler and validated with magnetic resonance in the carotid in a small volunteer population [31].

For both tissue and flow estimations, only a few groups have worked on their simultaneous assessment due to the technical limitations. Such a study combines difficulties from both tissue and flow imaging. In 2006, Tortoli et al. [32] developed a system that avoided the need for the beam-to-flow angle estimation and that extracted wall deformation and WSR. In 2008, Hasegawa and Kanai [33] proposed a phase-tracking method for wall motion that was coupled to power Doppler for blood flow. Three years later, Luo and Konofagou [34] carried out a feasibility study using speckle tracking, for both wall and flow estimation in the mouse heart in vivo. More recently, Ekroll et al. [35] evaluated a quantitative angle-independent 2D vector velocity estimator for both wall motion and flow assessment, as evaluated on realistic carotid simulations and in-vivo carotids. In 2018, Fekkes et al. [36] extracted simultaneously the vascular strain and blood vector velocity at with both a high-frequency probe and a standard frequency probe in a phantom study. Then in 2019, Goudot et al. [37] used vector flow imaging in carotid stenosis to evaluate wall shear stress. Unfortunately, none of these studies used these wall motion and flow velocity estimates further to measure the carotid properties in a clinical context. However, in this field, the recent translation to the clinic of advanced ultrasound methods has shown great clinical potential [38]. Notable examples include tracking of myocardial motion together with vortex dynamics [39], development of a portable vector flow imaging scanner [40], and application of super-resolution to breast cancer evaluation [41]. Such a translation to the clinic also appears relevant for wall motion and vector flow in the arterial system.

The study presented in this article follows on from two previous studies that used the TO technique for simultaneous wall motion and flow estimation on phantoms [26], and in-vivo carotids from volunteers [27]. The aim of the present study is to now demonstrate the clinical feasibility of simultaneous vessel wall motion and flow vector velocity measurements and their potential interest through marker evaluation.

\section{MethodS}

\section{A. Healthy and patient volunteer populations}

Healthy volunteers were recruited along with volunteer patients from St. Olavs Hospital, Trondheim, Norway. The study received approval from the Research Ethics Board and written consent was obtained from the volunteers for the scanning. Their carotids were recorded using a research ultrasound scanner (Vantage $^{\mathrm{TM}} 256$ system; Verasonics, Redmond, Washington, USA) with a linear probe array (9L-D; General Electric Company, Boston, Massachusetts, USA), following standard clinical routine examinations using an ultrasound system (Vivid ${ }^{\mathrm{TM}}$ E95; General Electric Company, Boston, Massachusetts, USA). All of these acquisitions were performed in the hospital by cardiologists. Prior to the imaging, thermal and acoustical measurements were taken to estimate the thermal and mechanical indices, in addition to the heating of the transducer surface. These indices were updated and shown in real time on the scanner during the imaging for the navigation acquisition sequence. All of the measurements are within the limits set by the international standard IEC 60601-2-37:2004 [42] (measurements: $\mathrm{MI}=0.375$, TIS $=2.961, \mathrm{TIB}=5.91$, TIC $=$ $5.91, \mathrm{P}=216.8 \mathrm{~mW}$, ISPTA $=382 \mathrm{~mW} / \mathrm{cm}^{2}, \Delta \mathrm{T}=0.23{ }^{\circ} \mathrm{C}$ ).

Common carotid arteries were imaged in the healthy volunteers (12 individuals: 10 males $/ 2$ females) and patient 
TABLE I

ACQUISITION PARAMETERS

\begin{tabular}{cc}
\hline Parameter & Value \\
\hline Probe & $9 \mathrm{~L}-\mathrm{D}, \mathrm{GE}$ \\
Number of elements & $192 \mathrm{elements}$ \\
Pitch & $229 \mu \mathrm{m}$ \\
Speed of sound & $1540 \mathrm{~m} / \mathrm{s}$ \\
\hline Transmission frequency & $4.8 \mathrm{MHz}$ \\
Sampling frequency & $19.2 \mathrm{MHz}$ \\
Transmission pulse & $2.5 \mathrm{cycles}$ \\
$P R F$ & $12000 \mathrm{~Hz}$ \\
Steering angle & {$\left[-15^{\circ} ; 15^{\circ}\right]$} \\
Transmission/receiving apodization & Rectangular \\
\hline Acquisition duration & $1.5 \mathrm{~s}$ \\
Compounding & $2 \mathrm{plane}$ waves \\
Images per second & 6000 \\
Beamforming & DAS, $F_{\#}=1.5$ \\
\hline
\end{tabular}

volunteers (12 individuals: 6 males/6 females); an additional case of stenosis in an internal carotid artery (male) is also reported. The carotids on both sides were scanned once during the examinations, for $1.5 \mathrm{~s}$ each. As the geometry and severity of disease can vary from one side to the other, the two carotids from each volunteer are considered as different datasets for the analysis. From the 24 common carotid arteries of the patients, three were out of plane, and two had strong clutter, so these were excluded from further analysis. All of the other acquisitions were suitable for analysis (from healthy volunteers and the additional case). The volunteer patients (19 carotids: 10 males/9 females) were subdivided into two categories: those without any apparent disease (13 carotids: 9 males/4 females) and those with apparent disease (6 carotids: 1 male/5 females), as diagnosed by the cardiologists during the clinical routine with the medical scanner, or based on their medical history. In the pathological subgroup, two carotids are from a patient who had had a stroke, one had a stenosis localized later (in the internal carotid), two had plaques, and one was greatly calcified. The healthy volunteers (24 carotids: 20 males/4 females) were arbitrarily separated into two subgroups: younger than 35 years old (18 carotids: $14 \mathrm{M} / 4 \mathrm{~F})$ or 35 years old or older (6 carotids: 6 males $/ 0$ females).

To achieve a high frame rate $(6000 \mathrm{~Hz})$ with a large field of view, two plane-wave acquisition schemes were used and summed after the beamforming, to increase the signal-to-noise ratio prior to estimation. The acquisition parameters are summarized in Table I. The channel data were recorded from the research scanner, after which the beamforming and processing were performed off-line using MATLAB (The MathWorks, Natick, Massachusetts, USA) by author V.P.. All of the measurements and potential markers were extracted using the same dataset (i.e., without sequential acquisition).

\section{B. Tissue motion estimation}

For wall displacement, an axial phase-based estimator was used. This is based on the phase measurement of the complex
TABLE II

MOTION ESTIMATION PARAMETERS

\begin{tabular}{ccc}
\hline Parameter & Tissue & Flow \\
\hline Lag & 12 frames $(2 \mathrm{~ms})$ & 1 frame $(0.17 \mathrm{~ms})$ \\
Ensemble length & 1 frame $(0.17 \mathrm{~ms})$ & 60 frames $(10 \mathrm{~ms})$ \\
Spatial window & Hann & Rectangular \\
Spatial window size $(x \times z)$ & $2 \mathrm{~mm} \times 1 \mathrm{~mm}$ & $2 \mathrm{~mm} \times 1 \mathrm{~mm}$ \\
TO wavelength & N/A & $0.8 \mathrm{~mm}$ \\
FWHM of TO & N/A & $2.0 \mathrm{~mm}$ \\
\hline
\end{tabular}

autocorrelation of the received signals, as originally proposed by Kasai et al. [43]:

$$
v_{z}=\frac{P R F}{\tau} \frac{c}{4 f_{c}} \frac{\Delta \hat{\Phi}(\tau)}{\pi}
$$

where $v_{z}$ is the axial component of the velocity, $P R F$ is the pulse repetition frequency, $\tau$ is the lag used for the estimation of the phase shift $\Delta \hat{\Phi}, c$ is the speed of sound, and $f_{c}$ is the center frequency. The left side of the equation does not depend on the lag as long as there is no aliasing and on the assumption of constant velocity in the observation window. The initial wall position (based on the first frame) is manually segmented, after which the wall is automatically tracked along the axial axis using the estimator described above.

Motion estimation is performed using spatial filtering onto the lag $\tau$ autocorrelation estimate in the complex domain, to reduce the variance of the estimates. The motion estimation parameters used in this study are summarized in Table II.

\section{Temporal adaptive clutter filtering}

Before flow estimation, the clutter signals need to be attenuated. In this study, finite impulse response filters were used, as they are numerically stable and as their cut-off is directly proportional to the tissue velocities. Instead of using one cut-off velocity for each acquisition, a temporal adaptive approach was used [44]. For each frame, a cut-off velocity was calculated based on the maximum estimated tissue velocity in that frame. As tissue velocities vary through the cardiac cycle, filters with a temporal adaptive cut-off velocity can achieve better performance than non-adaptive filters [35], [45]. In this study, we used finite impulse response equiripple filters of order 200, stopband attenuation of $70 \mathrm{~dB}$, and cut-off frequencies corresponding to twice the maximum tissue velocity in each frame.

\section{Flow estimation}

For 2D flow estimation, the TO approach was used. This approach is one way to separate the Doppler shift along two different virtual receiving apertures, related to two virtual receiving angles, which can be implemented in the post-processing. The main advantage of this post-processing approach is that the subapertures can be chosen retrospectively to avoid aliasing in the estimates. These two receiving subapertures create a lateral oscillation in the image that can 
be set.

Transverse oscillation is introduced by filtering in the Fourier domain [46] after clutter filtering. When using this method, the 2D Fourier spectrum of each ultrasound image is multiplied by a Gaussian function, to isolate the signal from the desired lateral frequency. This function is defined by the wavelength of the lateral oscillation and the full width at half-maximum (FWHM). After filtering, the $\mathrm{f}-\mathrm{k}$ spectrum contains four signal regions due to the natural axial oscillation of the radio-frequency images and the lateral oscillation introduced. Once the signals contain a 2D oscillation, additional filters are applied to produce two analytic signals, each with a frequency content from a single quadrant in the Fourier domain (Hann's approach) [47]. Each of these analytic signals corresponds to one of the two subapertures. This process implicitly assumes that the 2D oscillation can be described as the product of two 1D oscillation signals. The phase shift between temporal frames is then estimated for each of the analytic signals using the autocorrelation estimator. After phase estimation, the corresponding axial and lateral velocities are calculated using the relations:

$$
\left\{\begin{aligned}
v_{z} & =\frac{P R F}{\tau} \frac{c}{4 f_{c}} \frac{\Delta \hat{\Phi}^{I}(\tau)+\Delta \hat{\Phi}^{I I}(\tau)}{\pi} \\
v_{x} & =\frac{P R F}{\tau} \frac{c}{4 f_{x}} \frac{\Delta \hat{\Phi}^{I}(\tau)-\Delta \hat{\Phi}^{I I}(\tau)}{\pi}
\end{aligned}\right.
$$

where $\Delta \hat{\Phi}^{I}$ and $\Delta \hat{\Phi}^{I I}$ are the phase shifts calculated from the first and second quadrants, respectively, and $f_{x}$ is the lateral frequency introduced during filtering for the TO introduction.

Flow estimation is performed using spatial filtering onto the lag $\tau$ autocorrelation estimate in the complex domain, to reduce the variance of the estimates. The motion estimation parameters used in this study are summarized in Table II.

\section{E. Carotid properties: standard measurements}

As previously explained, the development of cardiovascular diseases influences the flow field as well as the properties of the vessel wall. After motion estimation, several characteristics can be extracted. The quantitative parameters that are estimated in this study based on tissue and flow estimates are described above.

From the wall velocity estimates:

- Wall velocity range $\left(\Delta V_{\text {wall }}, \mathrm{mm} / \mathrm{s}\right)$

- Mean diameter $(\bar{d}, \mathrm{~mm})$

- Arterial strain $(S, \%)$

From the flow velocity estimates:

- Peak systolic velocity $(P S V, \mathrm{~m} / \mathrm{s})$

- Resistivity index $(R I, \%)$

- Backflow ( $B$, ratio of acquisitions with backflow in the group, \%)

The wall velocity range is the difference between the maximum and minimum wall velocities along both walls during the cardiac cycle. This value represents the deformation of the vessel itself due to mechanical waves and blood pressure. It is expected to decrease when the arterial wall thickness or stiffness increase. The mean diameter is averaged over several cardiac cycles and the entire field of view. Pathologies influence the artery geometry, and thus the artery diameter; remodeling that occurs with aging also contributes to modifications to this parameter [48]. The arterial strain is the relative difference between the largest and smallest diameter during the heart cycle [49]. This value represents the response of the artery to the stress (i.e., blood pressure); stiff and calcified arteries are less compliant than healthy ones, and patients admitted after a stroke show lower arterial strain on average (typical value lower than $5 \%$ ) [50].

Peak systolic velocity is averaged along the centerline of the lumen, equidistant from both of the walls, based on the vector velocity field. This value is directly related to the maximum blood flow rate. In the case of a narrowing upstream, this value increases; also, the overall remodeling and compliance of the arterial system influence this velocity. The resistivity index is the ratio between the end-diastolic velocity and the peak systolic velocity (PSV); the end-diastolic velocity and the PSV are averaged along the centerline of the lumen. The resistivity index is related to the vascular resistance of the downstream vascular bed, which is primarily used for stenosis assessment. In the case of atherosclerosis, it is affected by both upstream and downstream diseases. The resistivity index is a marker of atherosclerosis risk if it is higher than $80 \%$ in the renal arteries [51] and has been studied in the carotid artery as a potential marker [52]. The waveform can be mono or triphasic in arteries. Triphasic flow indicates a backflow during the cardiac cycles, which has been evaluated in the aorta already using vector flow imaging [53]. In a normal carotid artery, backflow should not occur. If observed, it indicates a malfunction of the blood circulation, and as such, an abnormal recirculation [49]. Backflow is detected if a negative velocity is estimated along the centerline of the lumen during the cardiac cycles.

\section{F. Carotid properties: advanced measurements}

Wall stiffness can be assessed through PWV, which is directly linked to the elasticity of the medium [4]. The PWV can be estimated based on the wall acceleration maps at the dicrotic notch, which corresponds to the closure of the aortic valve [54]. For each spatial position in the wall, the velocity estimates are differentiated over time to estimate the wall acceleration. Figure 1 illustrates this for one spatial point in the wall during a cardiac cycle. Two peaks can be observed in Figure 1. The first peak (around $50 \mathrm{~ms}$ ) corresponds to a mechanical wave after the opening of the aortic valve, whereas the second peak (around $300 \mathrm{~ms}$ ) corresponds to another mechanical wave that occurs at the closure of the aortic valve, where the PWV can be extracted. For each point along the vessel, the passage of the pulse wave is defined as the midpoint between the two time points in which the 
(a)

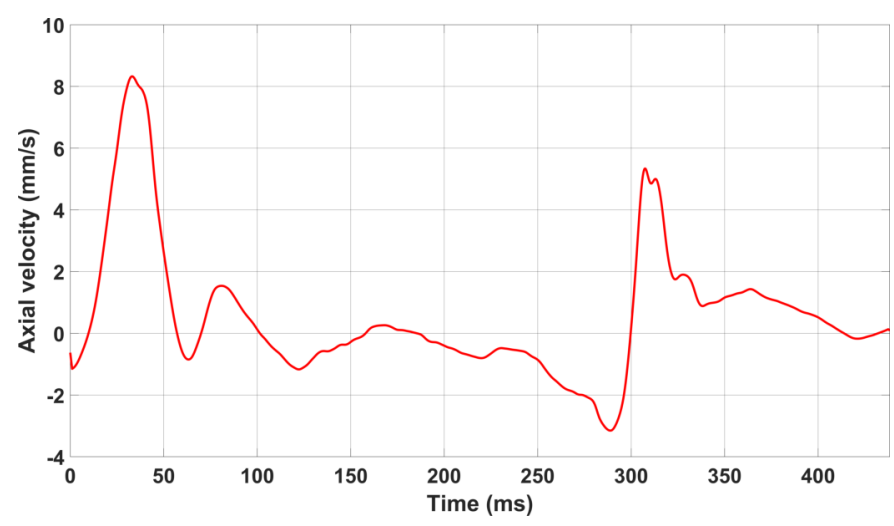

(b)

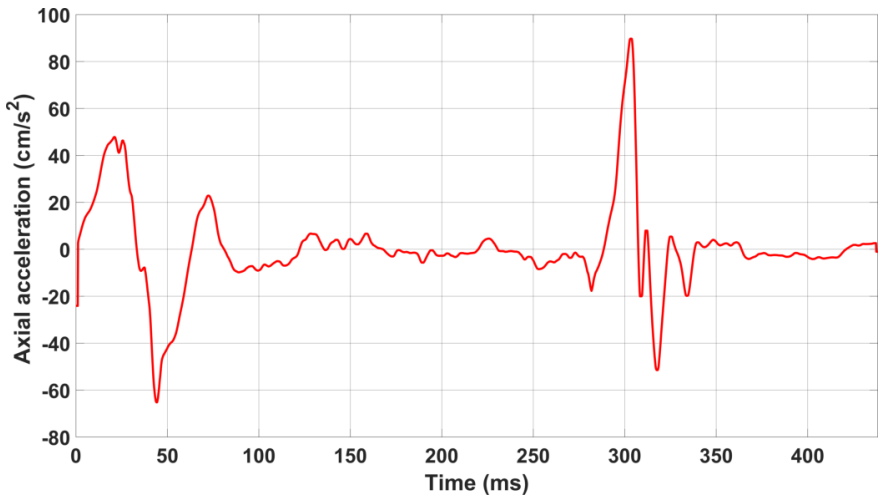

Fig. 1. (a) Temporal evolution of the wall velocity $(\mathrm{mm} / \mathrm{s})$ for a point in the upper wall. (b) Acceleration $\left(\mathrm{cm} / \mathrm{s}^{2}\right)$ computed over time based on (a). The peak around $300 \mathrm{~ms}$ corresponds to the dicrotic notch for the PWV estimation.

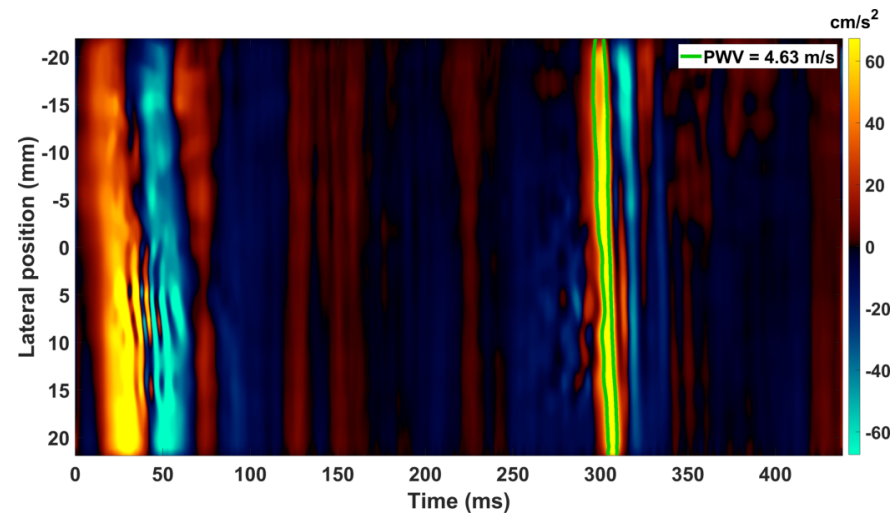

Fig. 2. Acceleration map $\left(\mathrm{cm} / \mathrm{s}^{2}\right)$ along the upper wall for a patient volunteer. The PWV is estimated at the dicrotic notch (closure of aortic valve, acceleration peak at $300 \mathrm{~ms}$ ), as defined at the midpoint of the half-maximum. acceleration is half the maximum value at the dicrotic notch; these midpoints are used instead of the maxima because they are less sensitive to noise. The maximum is roughly at $300 \mathrm{~ms}$ in Figure 1b. The temporal position of the peak is different for each position along the wall, due to the propagation of the pulse wave. Thus, the PWV can be estimated by using linear regression to estimate the propagation speed of the peak acceleration. An example is provided in Figure 2, with the bandwidth at half-maximum shown at around $300 \mathrm{~ms}$. For each carotid, the final PWV value is the average of the measurements on the upper and distant walls, using as many cardiac cycles as possible (typically from 1 to 3 ).

Another characteristic is the wall shear rate (WSR), which provides indirect information on the shear forces applied by the flow on the vessel wall. It has been shown that a low WSR can facilitate the development of plaques, whereas a high WSR after plaque development can increase the risk of inflammation and rupture [13]. This marker can be extracted from the vector flow maps as follows, knowing the position and orientation of the wall [55]:

$$
W S R=\left.\frac{\partial v}{\partial n}\right|_{\text {wall }}
$$

where $v$ is the 2D flow velocity, and $n$ is the wall normal. In this study, the WSR is evaluated for each patient $1 \mathrm{~mm}$ away from the upper and lower walls, over time. The mean WSR (mWSR) and the peak WSR (pWSR) are extracted as potential indicators. While the WSR is calculated along both walls without averaging, the mWSR is averaged over the cardiac cycles, and the pWSR is the maximum WSR detected over the cardiac cycles.

To sum up here, the advanced measurements are:

- Pulse wave velocity $(P W V, \mathrm{~m} / \mathrm{s})$

- Mean wall shear rate $\left(m W S R, s^{-1}\right)$

- Peak wall shear rate $\left(p W S R, s^{-1}\right)$

\section{G. Statistical hypothesis tests}

The statistical tests were performed using $\mathrm{R}$ 3.6.1 [56]. Except for the backflow, a permutation test was used. This statistical test builds sampling distributions for each group combination by resampling the observed data by permutations and reassigning each observation to a group. Then the experimental difference between one group and another can be evaluated on this permutation distribution, which is normal.

For the backflow, because this parameter is a categorical variable for each measurement, the p-values cannot be evaluated using the previous test. To compute the p-values in such a case, Fisher's exact tests were used. This statistical test is based on the analysis of a contingency table.

\section{RESULTS}

\section{A. Flow and tissue visualization}

Due to the simultaneous wall and flow velocity measurements, it becomes possible to study and visualize 
(a)

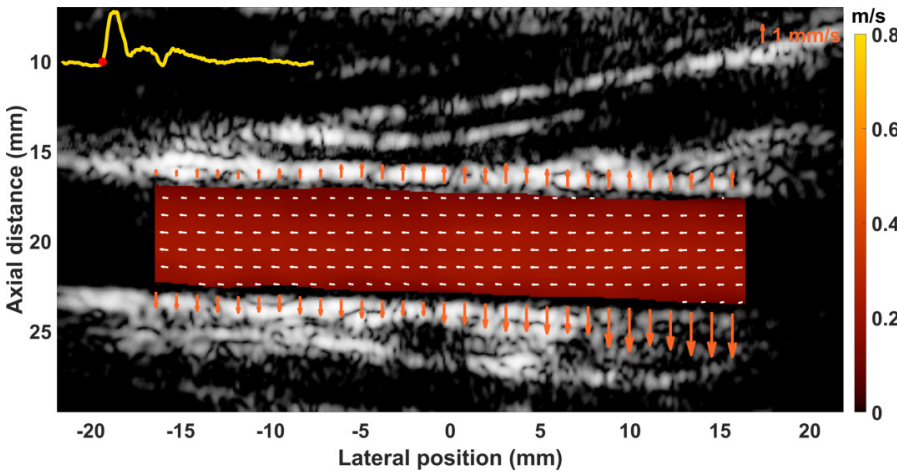

(c)

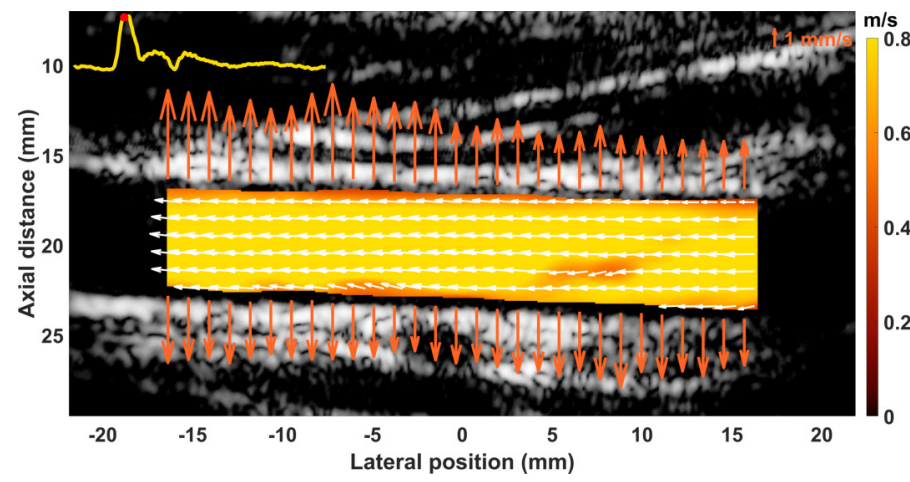

(e)

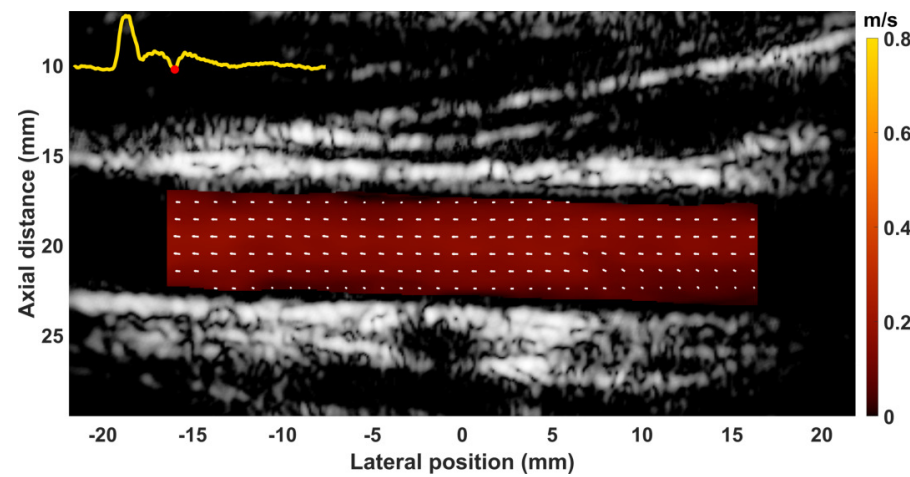

(b)

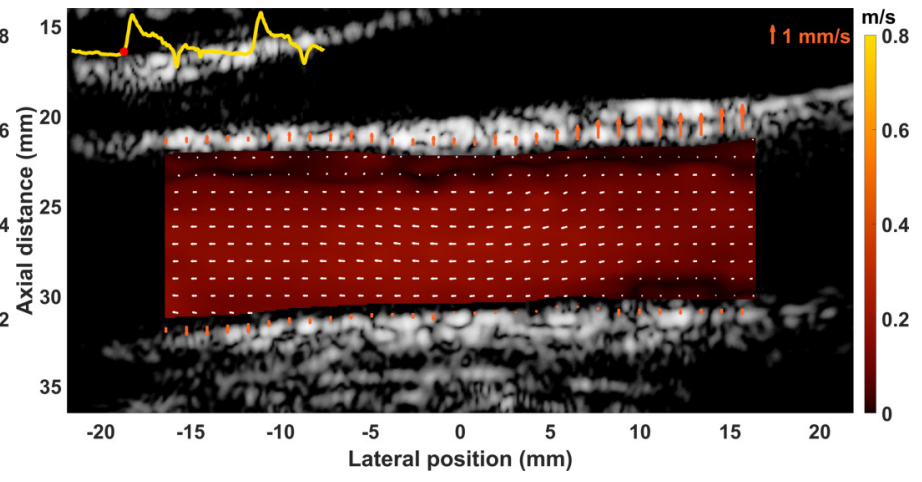

(d)

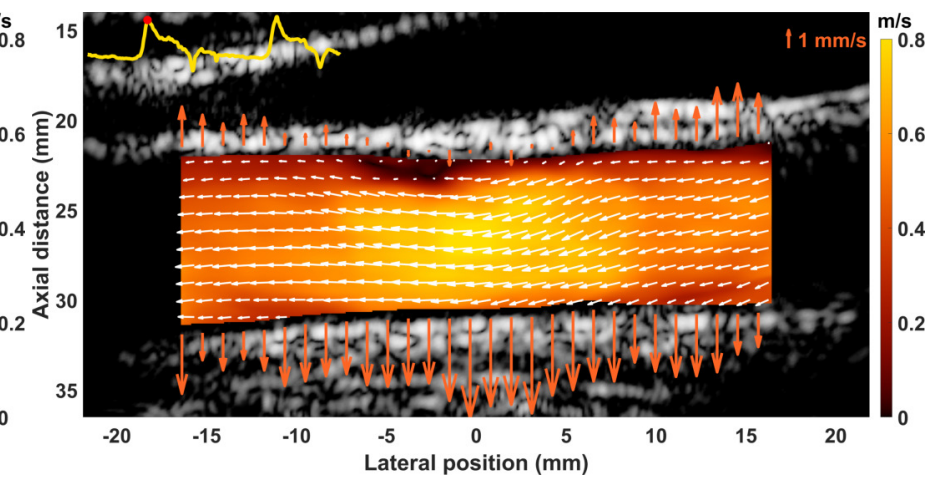

(f)

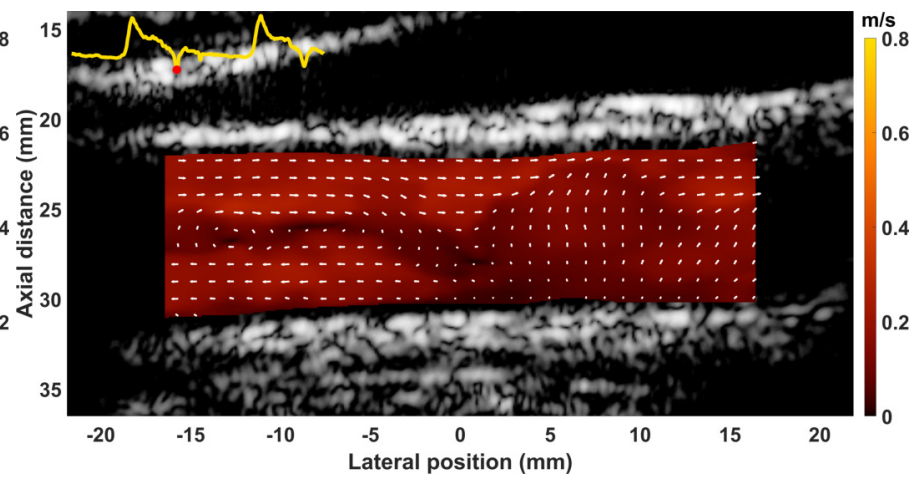

Fig. 3. Tissue motion and flow velocity estimates superimposed with B-mode images for a healthy volunteer (a, c, e) and a patient volunteer (b, d, f) at three different times: at the start of systole (a, b); at the systolic peak (c, d); and at the end of systole (closure of aortic valve) (e, f). The top-left plot in each image is the lateral flow velocity in the center of the lumen, and the top-right plot is the scale for the wall velocity $(\mathrm{mm} / \mathrm{s}$ ). For the flow, the colormap gives the magnitude of the flow $(\mathrm{m} / \mathrm{s})$, and the arrows represent both orientation and magnitude. For (e, f), the arrows are not shown for better flow visualization, because the vessel is in an active contracting phase and the arrows will overlap with the flow estimates. The carotids were recorded with the brain to the left of the images.

both of these characteristics at the same time. An example of the visualization is shown in Figure 3. For one of the healthy volunteers (Figure 3a, b, c), the flow is mainly parabolic (qualitative inspection) during the cardiac cycle, without any backflow or unusual flow pattern, and the walls move in correspondence to the cardiac cycle. For one of the patient volunteers (Figure 3d, e, f) who was admitted following a stroke coupled with plaques and calcifications, the radius of the artery is larger compared to the healthy volunteer. The flow shows an unusual pattern at the systolic peak (Figure 3e) close to the upper wall, with a low flow region; the wall was not moving around this region, and the flow was globally lower compared to the healthy volunteer. At the end of systole (Figure 3f), a complex flow pattern occurred, with backflow in the carotid, and a vortex is visible close to the carotid bifurcation (left of the image).

\section{B. Carotid properties}

The groups were compared in terms of the tissue estimates (Figure 4), flow measurements (Figure 5), and carotid properties (Figure 6). Table III summarizes all of the results as means and standard deviations, while the p-values for all of the possible combinations of groups and sub-groups are 
given in Table IV.

The wall velocity range ( $\Delta V_{\text {wall }}$, Figure $\left.4 \mathrm{a}\right)$ decreases with increased age and from the healthy volunteers to the patient volunteers. Furthermore, the wall velocity range separates the young healthy volunteers from the older healthy volunteers ( $\mathrm{p}$-value $\leq 0.01)$, and the healthy volunteers from the patient volunteers ( $\mathrm{p}$-value $\leq 0.001$ ). The artery diameter $(\bar{d}$, Figure $4 \mathrm{~b})$ is significantly greater for the patient volunteers than the healthy volunteers. The artery diameter separates both of these groups (p-value $\leq 0.001$ ); however, no significant differences are observed within the groups. The arterial strain ( $S$, Figure 4c) decreases with increased age and is lower for patient volunteers than for healthy volunteers, with significant differences between the young and older healthy volunteers ( $\mathrm{p}$-value $\leq 0.01$ ) and between the healthy volunteers and the patient volunteers ( $\mathrm{p}$-value $\leq 0.001)$.

Similar observations are found for the PSV (Figure 5a). Here, the older healthy volunteers have significantly lower PSV than the young healthy volunteers ( $\mathrm{p}$-value $\leq 0.01$ ), and the patient volunteers have significantly lower PSV than the healthy volunteers ( $\mathrm{p}$-value $\leq 0.001$ ). The resistivity index ( $R I$, Figure $5 \mathrm{~b}$ ) appears to decrease with increased age, and it separates young healthy volunteers from older healthy volunteers ( $\mathrm{p}$-value $\leq 0.01)$ and the two subgroups of patients ( $\mathrm{p}$-value $\leq 0.05$ ); however, it fails to separate the healthy volunteers from the patient volunteers. Backflow ( $B$, Figure 5c) is observed primarily in the patient volunteers with apparent pathologies. It is observed in $67 \%$ of these carotids, compared to less than $10 \%$ of all of the carotids in the other groups, with the difference between the two subgroups of patient volunteers significant ( $p$-value $\leq 0.05$ ); it also separates the healthy volunteers from the patient volunteers ( $\mathrm{p}$-value $\leq$ $0.05)$.

The PWV (Figure 6a) appears to increase with the risk. In this study, a value greater than $7 \mathrm{~m} / \mathrm{s}$ always corresponds to a patient volunteer with a diseased carotid. PWV separates the healthy volunteers from the patient volunteers ( $p$-value $\leq 0.01)$ and the older healthy volunteers from the patient volunteers with a pathology ( $\mathrm{p}$-value $\leq 0.05)$. The mWSR (Figure 6b) is lower for the patient volunteers than the healthy volunteers, with significant difference between these groups (p-value $\leq 0.001$ ). The pWSR (Figure 6c) is also higher for the healthy volunteers than for the patient volunteers, with a significant ( $p$-value $\leq 0.001$ ), and also significantly higher for young healthy volunteers compared to the older ones (p-value $\leq 0.01)$.

Repeatability was also evaluated across the cardiac cycles for the volunteers with at least three measurements of the same parameters (related to heart rate and the initial position of the recording in the cardiac cycle). All of the flow measurements have low standard deviations, with less than $2 \%$ for PSV for young healthy volunteers; for instance: PSV $=1.197 \mathrm{~m} / \mathrm{s}$ $\pm 0.6 \%$ (typical example). The standard deviations were less (a)

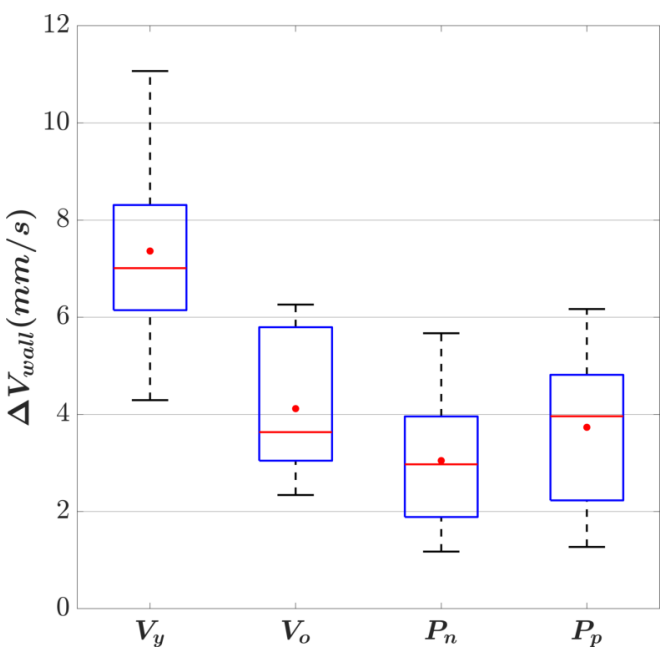

(b)

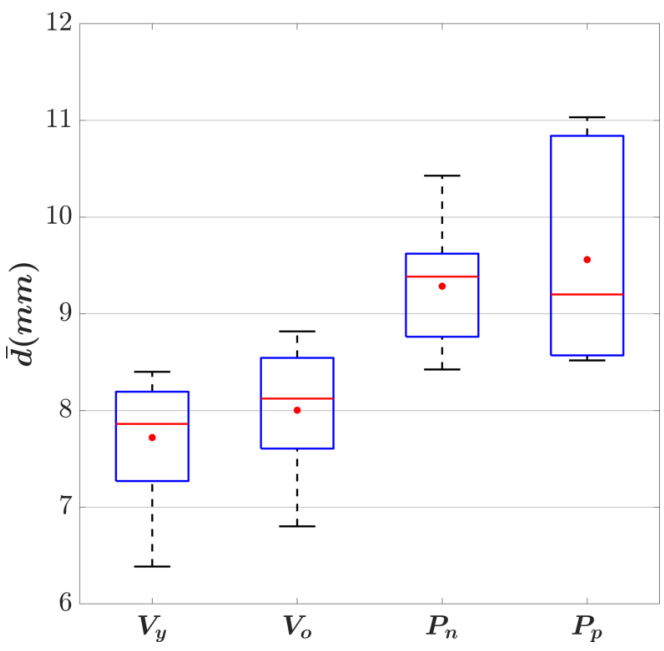

(c)

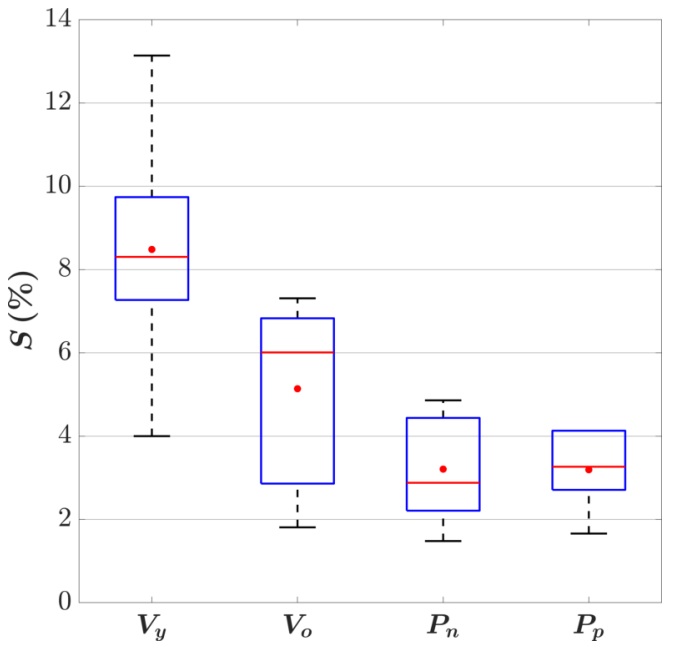

Fig. 4. Wall velocity range $\left(\Delta V_{\text {wall }}\right)$ (a), diameter of the artery $(\bar{d})(\mathrm{b})$, and arterial strain $(S)(\mathrm{c}) . \mathrm{V}$ and $\mathrm{P}$ indicate the healthy volunteers and patient volunteers; lower cases $\mathrm{Y}, \mathrm{O}, \mathrm{N}$, and $\mathrm{P}$ indicate the young, old, non-pathological, and pathological subgroups. 
TABLE III

METRICS EXTRACTED FROM THE HEALTHY AND PATIENT VOLUNTEER POPULATIONS

\begin{tabular}{clcccccc}
\hline & & \multicolumn{3}{c}{ Healthy volunteers } & \multicolumn{2}{c}{ Patients } \\
& & Young & Older & All & Non-pathological & Pathological & All \\
& Metrics & $\mu \pm \sigma$ & $\mu \pm \sigma$ & $\mu \pm \sigma$ & $\mu \pm \sigma$ & $\mu \pm \sigma$ & $\mu \pm \sigma$ \\
& & $N=18$ & $N=6$ & $N=24$ & $N=13$ & $N=6$ & $N=19$ \\
\hline \multirow{3}{*}{ Tissue } & $\Delta V_{\text {wall }}(\mathrm{mm} / \mathrm{s})$ & $7.36 \pm 1.89$ & $4.12 \pm 1.57$ & $6.55 \pm 2.29$ & $3.05 \pm 1.29$ & $3.73 \pm 1.79$ & $3.26 \pm 1.45$ \\
& $\bar{d}(\mathrm{~mm})$ & $7.72 \pm 0.58$ & $8.00 \pm 0.74$ & $7.80 \pm 0.62$ & $9.28 \pm 0.62$ & $9.56 \pm 1.12$ & $9.38 \pm 0.80$ \\
& $S(\%)$ & $8.48 \pm 2.44$ & $5.14 \pm 2.26$ & $7.65 \pm 2.77$ & $3.21 \pm 1.22$ & $3.19 \pm 0.93$ & $3.20 \pm 1.11$ \\
\hline \multirow{3}{*}{ Flow } & $P S V(\mathrm{~m} / \mathrm{s})$ & $1.15 \pm 0.16$ & $0.90 \pm 0.13$ & $1.09 \pm 0.19$ & $0.62 \pm 0.16$ & $0.67 \pm 0.20$ & $0.63 \pm 0.17$ \\
& $R I(\%)$ & $82.81 \pm 4.66$ & $74.83 \pm 6.66$ & $80.61 \pm 6.18$ & $79.56 \pm 3.76$ & $84.87 \pm 5.39$ & $81.24 \pm 4.89$ \\
& $B(\%)$ & $5.56 \pm N / A$ & $0 \pm N / A$ & $4.17 \pm N / A$ & $7.69 \pm N / A$ & $66.67 \pm N / A$ & $26.32 \pm N / A$ \\
\hline \multirow{3}{*}{ Advanced } & $P W V(\mathrm{~m} / \mathrm{s})$ & $4.03 \pm 0.98$ & $4.90 \pm 0.79$ & $4.26 \pm 1.00$ & $5.16 \pm 1.18$ & $7.26 \pm 1.94$ & $6.21 \pm 2.11$ \\
& $m W S R\left(\mathrm{~s}^{-1}\right)$ & $210 \pm 39$ & $227 \pm 25$ & $214 \pm 36$ & $158 \pm 42$ & $134 \pm 42$ & $150 \pm 42$ \\
& $p W S R\left(\mathrm{~s}^{-1}\right)$ & $1027 \pm 148$ & $784 \pm 121$ & $966 \pm 176$ & $521 \pm 137$ & $513 \pm 141$ & $519 \pm 134$ \\
\hline
\end{tabular}

TABLE IV

P-VALUES BETWEEN THE GROUPS AND SUBGROUPS DEPENDING ON THE METRIC. V AND P INDICATE THE HEALTHY VOLUNTEERS AND PATIENT VOLUNTEERS, RESPECTIVELY; LOWER CASES Y, O, N, AND P INDICATE THE YOUNG, OLD, NON-PATHOLOGICAL, AND PATHOLOGICAL SUBGROUPS, RESPECTIVELY

\begin{tabular}{|c|c|c|c|c|c|c|c|c|c|c|c|c|}
\hline \multicolumn{2}{|c|}{ Metrics } & \multirow{2}{*}{$\frac{\boldsymbol{V - P}}{<0.001}$} & \multirow{2}{*}{$\frac{\boldsymbol{V y}-\boldsymbol{V o}}{0.001}$} & \multirow{2}{*}{$\frac{\boldsymbol{P n}-\boldsymbol{P p}}{0.356}$} & \multirow{2}{*}{$\begin{array}{c}\boldsymbol{V y} \boldsymbol{y} \boldsymbol{P n} \\
<0.001\end{array}$} & \multirow{2}{*}{$\begin{array}{c}\boldsymbol{V y} \boldsymbol{y} \boldsymbol{P p} \\
<0.001\end{array}$} & \multirow{2}{*}{$\begin{array}{c}\text { Vo-Pn } \\
0.135\end{array}$} & \multirow{2}{*}{$\begin{array}{c}\boldsymbol{V o}-\boldsymbol{P p} \\
0.698\end{array}$} & \multirow{2}{*}{$\frac{\boldsymbol{V} \text {-Pn }}{<0.001}$} & \multirow{2}{*}{$\frac{\boldsymbol{V}-\boldsymbol{P p}}{0.009}$} & \multirow{2}{*}{$\begin{array}{c}\boldsymbol{P} \text {-Vy } \\
<0.001\end{array}$} & \multirow{2}{*}{$\begin{array}{c}\boldsymbol{P} \text {-Vo } \\
0.230\end{array}$} \\
\hline Tissue & $\Delta V_{\text {wall }}$ & & & & & & & & & & & \\
\hline IIssue & $\bar{d}$ & $<0.001$ & 0.340 & 0.499 & $<0.001$ & $<0.001$ & $<0.001$ & 0.012 & $<0.001$ & $<0.001$ & $<0.001$ & $<0.001$ \\
\hline & $S$ & $<0.001$ & 0.007 & 0.981 & $<0.001$ & $<0.001$ & 0.026 & 0.079 & $<0.001$ & $<0.001$ & $<0.001$ & 0.009 \\
\hline \multirow{3}{*}{ Flow } & $P S V$ & $<0.001$ & 0.002 & 0.528 & $<0.001$ & $<0.001$ & 0.002 & 0.027 & $<0.001$ & $<0.001$ & $<0.001$ & 0.002 \\
\hline & $R I$ & 0.814 & 0.003 & 0.023 & 0.049 & 0.376 & 0.057 & 0.013 & 0.518 & 0.154 & 0.323 & 0.017 \\
\hline & $B$ & 0.046 & 0.331 & 0.037 & 0.824 & 0.033 & 0.337 & 0.025 & 0.691 & 0.031 & 0.089 & 0.021 \\
\hline \multirow{3}{*}{ Advanced } & $P W V$ & 0.001 & 0.068 & 0.138 & 0.085 & $<0.001$ & 0.701 & 0.032 & 0.140 & $<0.001$ & 0.001 & 0.162 \\
\hline & $m W S R$ & $<0.001$ & 0.343 & 0.273 & 0.002 & $<0.001$ & 0.003 & 0.001 & $<0.001$ & $<0.001$ & $<0.001$ & $<0.001$ \\
\hline & $p W S R$ & $<0.001$ & 0.002 & 0.905 & $<0.001$ & $<0.001$ & 0.001 & 0.004 & $<0.001$ & $<0.001$ & $<0.001$ & $<0.001$ \\
\hline
\end{tabular}

than $10 \%$ for the pathological patient volunteers; for instance: PSV $=0.849 \mathrm{~m} / \mathrm{s} \pm 5.3 \%$ (typical example). The tissue measurements are also accurate, with less than $10 \%$ for all of the population; for instance: arterial strain: $\mathrm{S}=9.38 \%$ $\pm 9.5 \%$ (young healthy volunteer), $\mathrm{S}=3.24 \% \pm 8.36 \%$ (pathological patient volunteer). The PWV values are also repeatable through the cardiac cycles, with standard deviation of less than $10 \%$ : PWV $=3.60 \mathrm{~m} / \mathrm{s} \pm 3.0 \%$ (young healthy volunteer). Comparisons of the two carotids for the same volunteers show significant differences; for instance: PWV (right $/$ left $)=4.45 \mathrm{~m} / \mathrm{s} / 6.41 \mathrm{~m} / \mathrm{s}$ (older healthy volunteer, increase of $44.05 \%$ ); PWV (right / left) $=9.29 \mathrm{~m} / \mathrm{s} / 6.42 \mathrm{~m} / \mathrm{s}$ (patient, decrease of $30.89 \%$, right carotid with a plaque).

\section{Additional case (internal carotid stenosis)}

An additional example is included to show the potential extension and the feasibility of this method with more complex geometry and advanced diseased. This case is not included in the previous measurements as it is a different artery. Figure 7 shows an internal carotid artery of a patient with severe stenosis. In the stenotic region, the wall moved slower compared to the overall upper wall (Figure 7a). Moreover, the pWSR was greater at this position. Before the stenosis, the flow was parabolic (qualitative inspection), whereas after the stenosis, a jetstream is seen with potential out-of-plane flow surrounding the central jet (Figure 7b).

\section{DISCUSSION AND CONCLUSION}

The feasibility of simultaneous measurements of the vessel wall motion and the blood flow velocity was investigated here in a relevant clinical context and at high frame rates. The wall motion and the flow were extracted for all of the healthy volunteers. For the patient volunteers, excluding the out-of-plane acquisitions, the feasibility was around 90\%, which is relatively high; the remaining $10 \%$ is due to the strong clutter in the flow. Furthermore, advanced clinical markers such as PWV and WSR were investigated, and statistical analysis was performed by comparing the groups of healthy volunteers to the patient volunteers with different diseases.

The visualization of the tissue motion and flow shows similar results for all of the healthy volunteers, with uniform motion in the wall and a qualitative parabolic flow profile. In the patient volunteers, the resulting images show complex flow patterns, vortices, and low-velocity regions close to the wall. The vector velocity fields allow detailed analysis of the flow patterns compared to the conventional color Doppler imaging, where only one component of the velocity can be extracted. The technique can also be used for the detection of unusual wall motion. In this study, the method highlights motionless parts of the arterial wall.

Using the measured parameters, it was possible to separate 
(a)

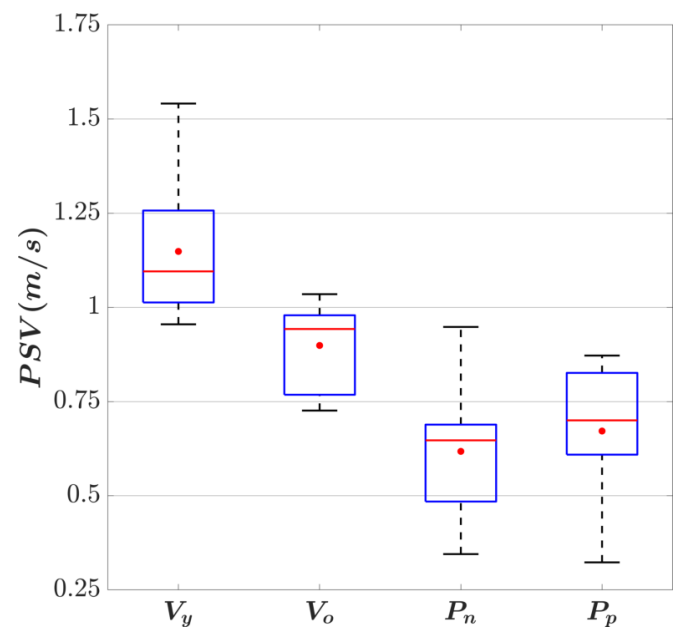

(b)

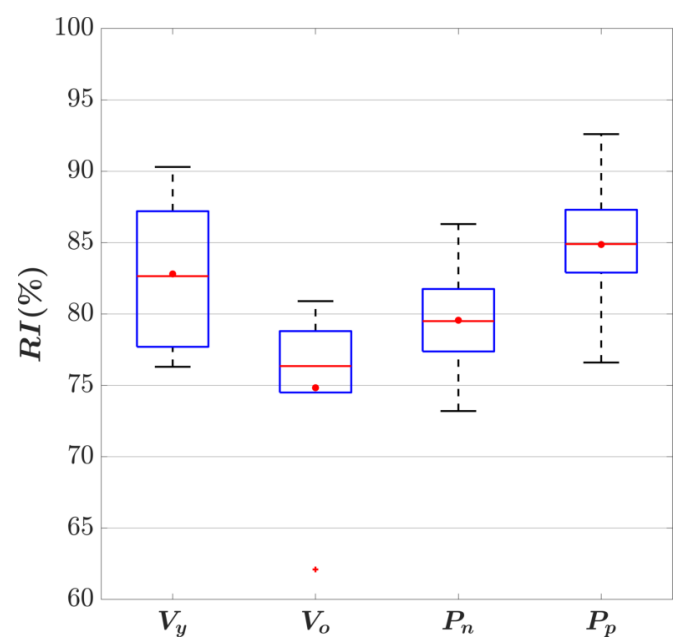

(c)

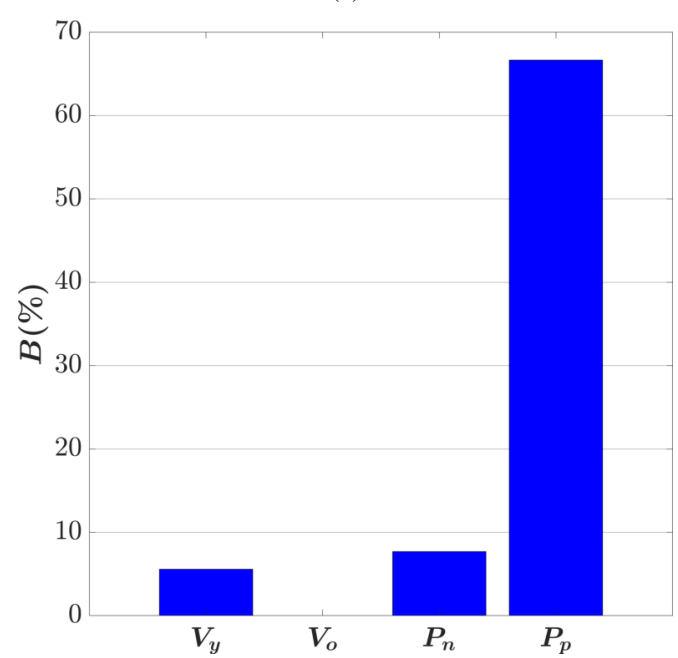

Fig. 5. Peak flow velocity $(P S V)$ (a), resistivity index $(R I)($ b), and backflow $(B)$ (c). $\mathrm{V}$ and $\mathrm{P}$ indicate the healthy volunteers and patient volunteers; lower cases $\mathrm{Y}, \mathrm{O}, \mathrm{N}$, and $\mathrm{P}$ indicate the young, old, non-pathological, and pathological subgroups. (a)

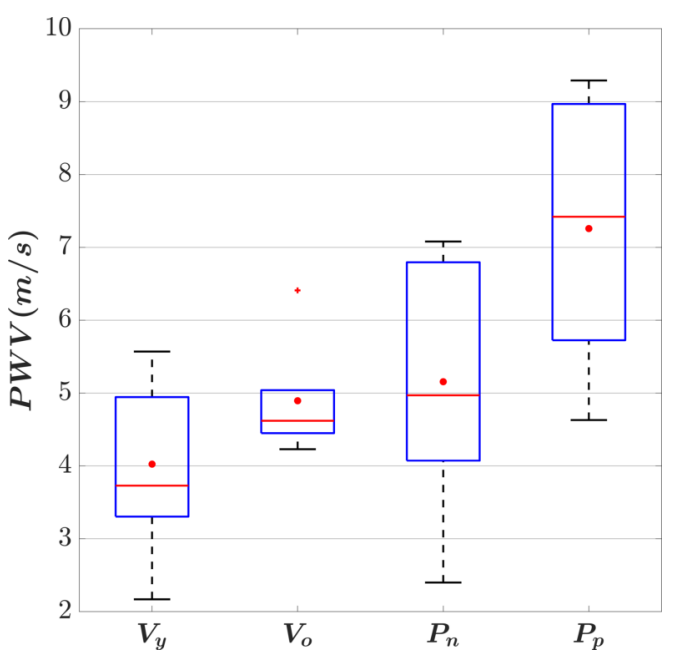

(b)

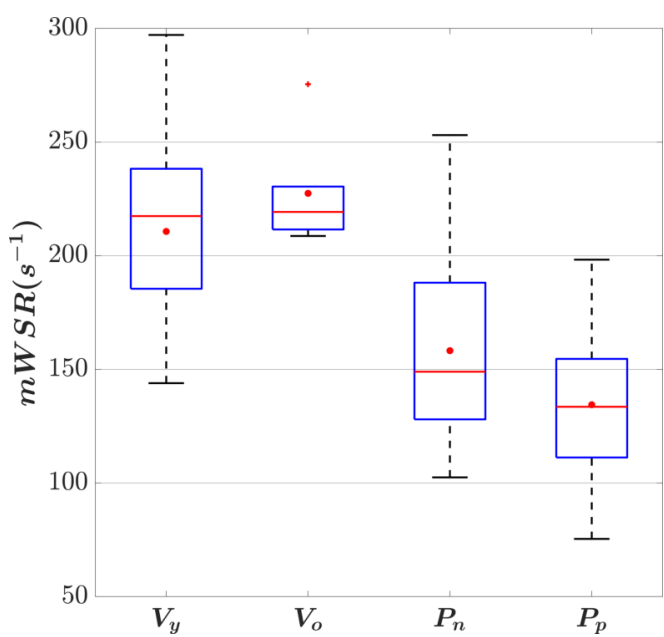

(c)

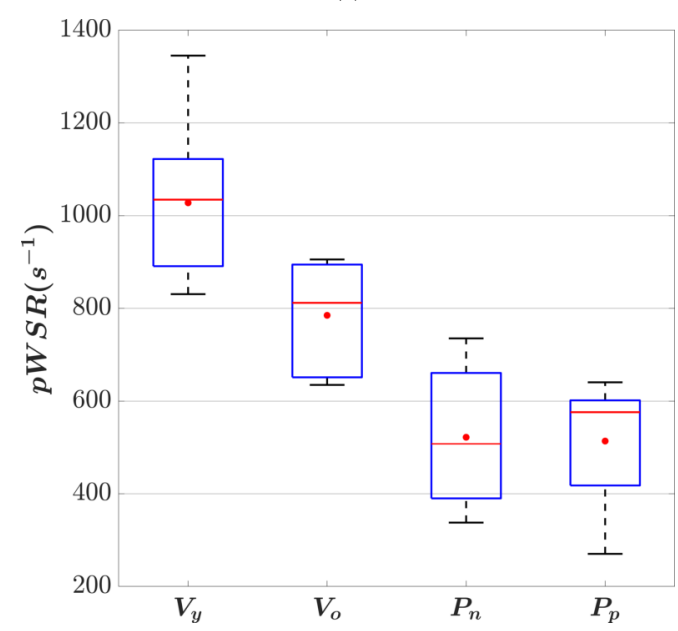

Fig. 6. Pulse wave velocity (PWV) (a), mean wall shear rate (mWSR) (b), and peak wall shear rate (pWSR) (c). V and P indicate the healthy volunteers and patients; lower cases $\mathrm{Y}, \mathrm{O}, \mathrm{N}$, and $\mathrm{P}$ indicate the young, old, non-pathological, and pathological subgroups. 
(a)

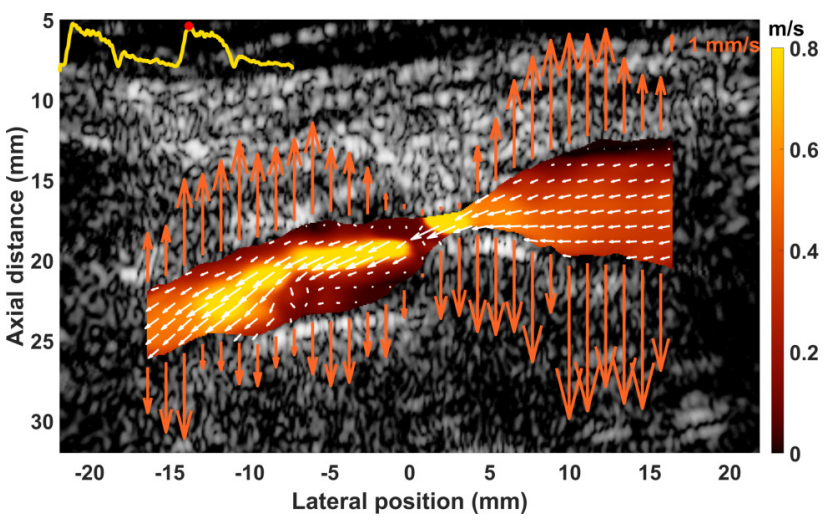

(b)

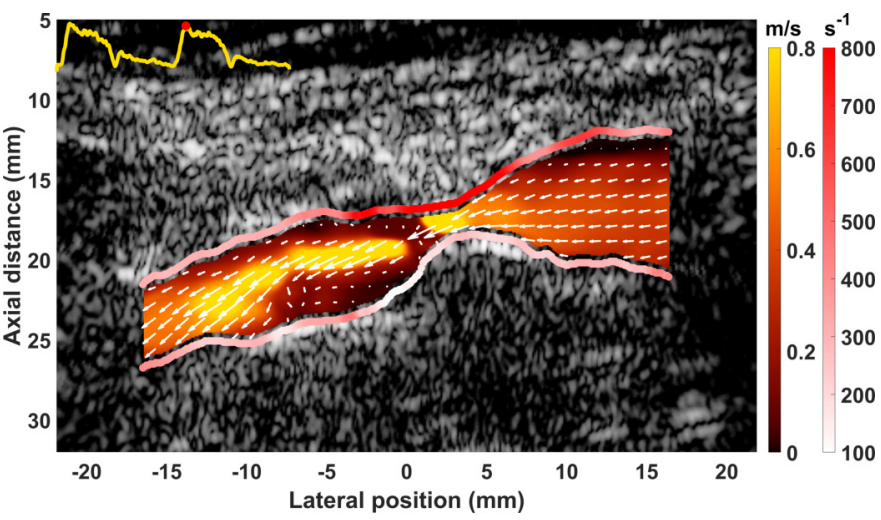

Fig. 7. Flow and tissue velocity estimates superimposed with the B-mode images for the internal carotid artery with stenosis (a). Tissue velocity is replaced with the local pWSR along the wall (b). The top-left plot in each image is the lateral flow velocity in the center of the lumen, and the top-right plot in (a) is the scale for the wall velocity $(\mathrm{mm} / \mathrm{s})$, while the colors in the wall in (b) represent the peak wall shear rates $\left(s^{-1}\right)$. For the flow, the colormap gives the magnitude of the flow $(\mathrm{m} / \mathrm{s})$, while the arrows represent both the orientation and the magnitude. The carotids were recorded with the brain to the left of the images.

the healthy volunteers from the patient volunteers, the young from the elderly, and the patient volunteers with an apparent disease from the others.

Several aspects can be defined as limitations here. First, due to the small number of patients, this study must be regarded as proof of concept, or a pilot study for transfer to the clinic, and not a full clinical study. The grouping was not disease-specific due to the limited number of patients, and it is not possible to correlate the different disease parameters as some diseases can have opposite behaviors. Such grouping could reveal more disease-specific behavior, such as higher PSV for stenosis or increased PWV for rigid plaques. In this study, the markers revealed overall trends for validation. Also, the grouping of the patients was based on the observations of the cardiologist during the conventional screening. However, this does not exclude undetected diseases in the arterial system or hypertension/ hypotension. This aspect might explain the difficulty in the separation of the two subgroups of patients and the variability in the measurements for the two subgroups. Some missing histories might also lead to bias for the patients.
Even if most of the computation is automatic here, inter-observer or intra-observer variation might lead to different measurements, and this should be estimated in any extended study. Moreover, the out-of-plane motion might affect the estimates, and aligning of the probe to the carotid geometry is challenging, especially in the patient population. The $2 \mathrm{D}$ tracking of the wall was impossible in the patient population due to noisy and probably inaccurate lateral tracking, which resulted in the use of an axial velocity estimator; out-of-plane motion is the possible explanation here. Moreover, this limitation is certainly at the origin of errors in the measurements of the WSR for the complex flow that is not in the scan plane. A solution might be to use a $3 \mathrm{D}$ imaging technique for both the tissue and flow motion estimations. Unfortunately, 3D imaging is still challenging in vivo, especially for the extraction of vector velocity fields in the flow. However, significant progress has already been made, as demonstrated by Papadacci et al. [57] in a feasibility study of color and tissue Doppler with a single beat in the left ventricle. Finally, the WSR was measured in this study, and not the wall shear stress. Although the stress due to the passing blood flow would be better represented by the wall shear stress, this value is dependent on the value of the blood viscosity ( $W S S=\mu W S R$, where $\mu$ is the viscosity of the flow), which can change locally in the arterial system. We also limited our study to nine potential markers, although many other potential markers exist, such as, for instance, vortography [58], flow complexity [59], pulse wave extracted from the flow, or longitudinal motion in the wall [60]. The increase in the number of markers would make the analysis challenging. One possible solution would be the use of artificial intelligence to analyze all of the markers, and also to automatically segment the lumen, as for cardiac imaging [61].

Overall, this study shows the feasibility of the investigated methods in a clinical context using a research scanner. The velocity-derived measurements were successfully extracted for both the healthy volunteers and the relevant patient volunteer population, including complex geometries with stenosis and remodeling. Future studies should include more carotids with different profiles, to specifically evaluate the potential clinical benefits. The next step will be a longitudinal study to determine whether the combination of wall motion and flow estimates will lead to better detection of high-risk profiles and early detection of plaques, and will assist the clinician in the vulnerability assessment of plaques, to decide whether surgical treatment is appropriate. Depending on the benefits, closer collaborations with clinicians must be established to assess the feasibility of the potential application of this technique to the clinic.

\section{REFERENCES}

[1] S. Satomura, "Ultrasonic doppler method for the inspection of cardiac functions," The Journal of the Acoustical Society of America, vol. 29, no. 11, pp. 1181-1185, nov 1957.

[2] B. Sigel, "A brief history of doppler ultrasound in the diagnosis of peripheral vascular disease," Ultrasound in Medicine \& Biology, vol. 24, no. 2, pp. 169-176, feb 1998. 
[3] A. Thrush and T. Hartshorne, Vascular Ultrasound: How, Why and When. Churchill Livingstone, 2009.

[4] K. Fujikura et al., "A novel noninvasive technique for pulse-wave imaging and characterization of clinically-significant vascular mechanical properties in vivo," Ultrasonic Imaging, vol. 29, no. 3, pp. 137-154, jul 2007.

[5] Reference Values for Arterial Stiffness' Collaboration, "Determinants of pulse wave velocity in healthy people and in the presence of cardiovascular risk factors: "establishing normal and reference values'," European Heart Journal, vol. 31, no. 19, pp. 2338-2350, jun 2010.

[6] A. I. Moens, Die Pulscurve. Leiden: E.J. Brill, 1878.

[7] D. H. Bergel, "The dynamic elastic properties of the arterial wall," The Journal of Physiology, vol. 156, no. 3, pp. 458-469, may 1961.

[8] S. Laurent et al., "Aortic stiffness is an independent predictor of all-cause and cardiovascular mortality in hypertensive patients," Hypertension, vol. 37, no. 5, pp. 1236-1241, 2001.

[9] K. Sutton-Tyrrell et al., "Elevated aortic pulse wave velocity, a marker of arterial stiffness, predicts cardiovascular events in well-functioning older adults," Circulation, vol. 111, no. 25, pp. 3384-3390, Jun. 2005.

[10] M. F. O'Rourke et al., "Clinical applications of arterial stiffness; definitions and reference values," American Journal of Hypertension, vol. 15 , no. 5, pp. 426-444, 052002.

[11] J. Luo, R. X. Li, and E. E. Konofagou, "Pulse wave imaging of the human carotid artery: an in vivo feasibility study," IEEE Transactions on Ultrasonics, Ferroelectrics and Frequency Control, vol. 59, no. 1, pp. 174-181, jan 2012.

[12] R. X. Li et al., "Pulse wave imaging in normal, hypertensive and aneurysmal human aortas in vivo: a feasibility study," Physics in medicine and biology, vol. 58, no. 13, p. 4549-4562, July 2013.

[13] J. Ohayon et al., "Biomechanics of atherosclerotic coronary plaque: Site, stability and in vivo elasticity modeling," Annals of Biomedical Engineering, vol. 42, no. 2, pp. 269-279, sep 2013.

[14] A. Lafont, "Basic aspects of plaque vulnerability," Heart, vol. 89, no. 10, pp. 1262-1267, oct 2003.

[15] E. G. Grant et al., "Carotid artery stenosis: Gray-scale and doppler US diagnosis-society of radiologists in ultrasound consensus conference," Radiology, vol. 229, no. 2, pp. 340-346, nov 2003.

[16] C. Wittens et al., "Editor's choice - management of chronic venous disease," European Journal of Vascular and Endovascular Surgery, vol. 49, no. 6, pp. 678-737, Jun. 2015.

[17] S. F. Stewart, "Effects of transducer, velocity, doppler angle, and instrument settings on the accuracy of color doppler ultrasound,' Ultrasound in Medicine \& Biology, vol. 27, no. 4, pp. 551-564, apr 2001.

[18] B. Dunmire et al., "Cross-beam vector doppler ultrasound for angle-independent velocity measurements," Ultrasound in Medicine \& Biology, vol. 26, no. 8, pp. 1213-1235, oct 2000.

[19] R. Steel and P. Fish, "Velocity bias and fluctuation in the standard dual beam doppler reconstruction algorithm," IEEE Transactions on Ultrasonics, Ferroelectrics and Frequency Control, vol. 49, no. 10, pp. $1375-1383$, oct 2002.

[20] V. L. Newhouse, L. W. Varner, and P. J. Bendick, "Geometrical spectrum broadening in ultrasonic doppler systems," IEEE Transactions on Biomedical Engineering, vol. BME-24, no. 5, pp. 478-480, sep 1977.

[21] J. Udesen et al., "High frame-rate blood vector velocity imaging using plane waves: Simulations and preliminary experiments," IEEE Transactions on Ultrasonics, Ferroelectrics and Frequency Control, vol. 55, no. 8, pp. 1729-1743, Aug. 2008.

[22] S. Ricci, L. Bassi, and P. Tortoli, "Real-time vector velocity assessment through multigate doppler and plane waves," IEEE Transactions on Ultrasonics, Ferroelectrics, and Frequency Control, vol. 61, no. 2, pp. 314-324, Feb. 2014.

[23] M. M. Pedersen et al., "Novel flow quantification of the carotid bulb and the common carotid artery with vector flow ultrasound," Ultrasound in Medicine \& Biology, vol. 40, no. 11, pp. 2700-2706, Nov. 2014.

[24] J. Jensen and P. Munk, "A new method for estimation of velocity vectors," IEEE Transactions on Ultrasonics, Ferroelectrics and Frequency Control, vol. 45, no. 3, pp. 837-851, may 1998.

[25] H. Liebgott et al., "Transverse oscillations for tissue motion estimation," Ultrasonics, vol. 50, no. 6, pp. 548-555, may 2010.

[26] V. Perrot et al., "Simultaneous pulse wave and flow estimation at high-framerate using plane wave and transverse oscillation on carotid phantom," in 2017 IEEE International Ultrasonics Symposium (IUS). IEEE, sep 2017.

[27] _ - "Simultaneous tissue and flow estimation at high frame rate using plane wave and transverse oscillation on in vivo carotid," in 2018 IEEE International Ultrasonics Symposium (IUS). IEEE, oct 2018.
[28] K. Hansen et al., "In vivo comparison of three ultrasound vector velocity techniques to MR phase contrast angiography," Ultrasonics, vol. 49, no. 8, pp. 659-667, Dec. 2009.

[29] J. Jensen et al., "Vector velocity volume flow estimation: Sources of error and corrections applied for arteriovenous fistulas," Ultrasonics, vol. 70, pp. 136-146, Aug. 2016.

[30] K. L. Hansen et al., "Vector flow imaging compared with conventional doppler ultrasound and thermodilution for estimation of blood flow in the ascending aorta," Ultrasonic Imaging, vol. 39, no. 1, pp. 3-18, Aug. 2016.

[31] A. H. Brandt et al., "A comparison study of vector velocity, spectral doppler and magnetic resonance of blood flow in the common carotid artery," Ultrasound in Medicine \& Biology, vol. 44, no. 8, pp. 1751-1761, Aug. 2018.

[32] P. Tortoli et al., "Noninvasive simultaneous assessment of wall shear rate and wall distension in carotid arteries," Ultrasound in Medicine \& Biology, vol. 32, no. 11, pp. 1661-1670, Nov. 2006

[33] H. Hasegawa and H. Kanai, "Simultaneous imaging of artery-wall strain and blood flow realized by high frame rate acquisition of RF echoes," in 2008 IEEE Ultrasonics Symposium. IEEE, nov 2008.

[34] J. Luo and E. E. Konofagou, "Imaging of wall motion coupled with blood flow velocity in the heart and vessels in vivo: A feasibility study," Ultrasound in Medicine \& Biology, vol. 37, no. 6, pp. 980-995, jun 2011.

[35] I. K. Ekroll et al., "Simultaneous quantification of flow and tissue velocities based on multi-angle plane wave imaging," IEEE Transactions on Ultrasonics, Ferroelectrics and Frequency Control, vol. 60, no. 4, pp. 727-738, apr 2013.

[36] S. Fekkes et al., "Simultaneous vascular strain and blood vector velocity imaging using high-frequency versus conventional-frequency plane wave ultrasound: A phantom study," IEEE Transactions on Ultrasonics, Ferroelectrics, and Frequency Control, vol. 65, no. 7, pp. 1166-1181, Jul. 2018.

[37] G. Goudot et al., "Assessment of wall shear stress by ultrafast vector flow imaging in carotid atheromatous stenosis," Archives of Cardiovascular Diseases Supplements, vol. 11, no. 1, pp. 96-97, Jan. 2019.

[38] C. M. Gallippi and J. J. Dahl, "Guest editorial special issue on pilot clinical translation of new medical ultrasound methodologies," IEEE Transactions on Ultrasonics, Ferroelectrics, and Frequency Control, vol. 66, no. 3, pp. 423-424, Mar. 2019.

[39] J. Faurie et al., "Coupling myocardium and vortex dynamics in diverging-wave echocardiography," IEEE Transactions on Ultrasonics, Ferroelectrics, and Frequency Control, vol. 66, no. 3, pp. 425-432, Mar. 2019.

[40] T. D. Ianni et al., "Portable vector flow imaging compared with spectral doppler ultrasonography," IEEE Transactions on Ultrasonics, Ferroelectrics, and Frequency Control, vol. 66, no. 3, pp. 453-462, Mar. 2019.

[41] S. Dencks et al., "Clinical pilot application of super-resolution US imaging in breast cancer," IEEE Transactions on Ultrasonics, Ferroelectrics, and Frequency Control, vol. 66, no. 3, pp. 517-526, Mar. 2019.

[42] "IEC 60601-2-37:2004 Medical electrical equipment - Part2-37: Particular requirements for the safety of ultrasonic medical diagnostic and monitoring equipment, Edition 1:2001 consolidated with amendment 1:2004. 1.1. International Electrotechnical Commission, 2004."

[43] C. Kasai et al., "Real-time two-dimensional blood flow imaging using an autocorrelation technique," IEEE Transactions on Sonics and Ultrasonics, vol. 32, no. 3, pp. 458-464, may 1985.

[44] I. K. Ekroll and J. Avdal, "Adaptive clutter filtering based on tissue vector velocities," in 2017 IEEE International Ultrasonics Symposium (IUS). IEEE, sep 2017.

[45] V. Perrot et al., "Spatial and temporal adaptive FIR clutter filtering," in 2018 IEEE International Ultrasonics Symposium (IUS). IEEE, oct 2018.

[46] S. Salles et al., "Plane wave transverse oscillation (PWTO): An ultra-fast transverse oscillation imaging mode performed in the fourier domain for 2d motion estimation of the carotid artery," in 2014 IEEE 11th International Symposium on Biomedical Imaging (ISBI). IEEE, apr 2014.

[47] T. Bulow and G. Sommer, "Hypercomplex signals-a novel extension of the analytic signal to the multidimensional case," IEEE Transactions on Signal Processing, vol. 49, no. 11, pp. 2844-2852, 2001.

[48] M. R. Ward et al., "Arterial remodeling," Circulation, vol. 102, no. 10, pp. 1186-1191, Sep. 2000 
[49] R. S. Reneman et al., "Flow velocity patterns in and distensibility of the carotid artery bulb in subjects of various ages." Circulation, vol. 71, no. 3, pp. 500-509, Mar. 1985.

[50] E. Y. Yang et al., "Carotid arterial wall characteristics are associated with incident ischemic stroke but not coronary heart disease in the atherosclerosis risk in communities (ARIC) study," Stroke, vol. 43, no. 1, pp. 103-108, Jan. 2012.

[51] M. E. Tublin, R. O. Bude, and J. F. Platt, "The resistive index in renal doppler sonography: Where do we stand?" American Journal of Roentgenology, vol. 180, no. 4, pp. 885-892, apr 2003.

[52] B. Frauchiger et al., "Comparison of carotid arterial resistive indices with intima-media thickness as sonographic markers of atherosclerosis," Stroke, vol. 32, no. 4, pp. 836-841, apr 2001.

[53] K. L. Hansen et al., "Analysis of systolic backflow and secondary helical blood flow in the ascending aorta using vector flow imaging," Ultrasound in Medicine \& Biology, vol. 42, no. 4, pp. 899-908, Apr. 2016.

[54] E. Hermeling et al., "The dicrotic notch as alternative time-reference point to measure local pulse wave velocity in the carotid artery by means of ultrasonography," Journal of Hypertension, vol. 27, no. 10, pp. 2028-2035, oct 2009.

[55] P. J. Brands et al., "A noninvasive method to estimate wall shear rate using ultrasound," Ultrasound in Medicine \& Biology, vol. 21, no. 2, pp. 171-185, Jan. 1995.

[56] $\mathrm{R}$ Development Core Team, R: A Language and Environment for Statistical Computing, R Foundation for Statistical Computing, Vienna, Austria, 2008, ISBN 3-900051-07-0. [Online]. Available: http://www.R-project.org

[57] C. Papadacci et al., "4D simultaneous tissue and blood flow doppler imaging: revisiting cardiac doppler index with single heart beat $4 \mathrm{~d}$ ultrafast echocardiography," Physics in Medicine \& Biology, vol. 64, no. 8, p. 085013 , Apr. 2019.

[58] F. Mehregan et al., "Doppler vortography: A color doppler approach to quantification of intraventricular blood flow vortices," Ultrasound in Medicine \& Biology, vol. 40, no. 1, pp. 210-221, jan 2014.

[59] K. Hansen et al., "Vector flow imaging compared with digital subtraction angiography for stenosis assessment in the superficial femoral artery - a study of vector concentration, velocity ratio and stenosis degree percentage," Ultrasound International Open, vol. 05, no. 02, pp. E53-E59, Mar. 2019.

[60] M. Cinthio et al., "Longitudinal movement of the common carotid artery wall: New information on cardiovascular aging," Ultrasound in Medicine \& Biology, vol. 44, no. 11, pp. 2283-2295, Nov. 2018.

[61] S. Leclerc et al., "Deep learning for segmentation using an open large-scale dataset in 2d echocardiography," IEEE Transactions on Medical Imaging, vol. 38, no. 9, pp. 2198-2210, Sep. 2019. 